\title{
Deformation analysis for the key structure of linear visual detection device
}

\author{
Junyi Hou ${ }^{1, a}$, Zailiang Chen ${ }^{1, b}$ and Zhenyu Chen ${ }^{2, c}$ \\ ${ }^{1}$ College of Mechanical and Electric Engineering, Soochow University, Suzhou 215021, China \\ ${ }^{2}$ College of Engineering, Michigan State University, East Lansing,USA \\ a346450300@qq.com, ${ }^{\text {b }}$ chenzailiang@suda.edu.cn, ${ }^{\text {c }}$ chenzhe6@msu.edu
}

Keywords: visual detection, camera, deflection, finite element simulation

\begin{abstract}
According to the different installation place of linear array camera component, the material property and mesh are set for the profiles which are fixed with camera by ANSYS Workbench. In the gravity of relevant parts, do the finite element simulation of deformation. According to the flexural deformation abstained by finite element analysis, use trimming device to adjust, So that the camera CCD plane and the table surface can maintain a certain degree of parallel, to ensure that the images captured by the linear array camera is not distorted, to improve the image quality and reduce the error of the measurement system.
\end{abstract}

\section{Introduction}

Due to the rapid development of optical, electronics, precision machinery and computer control technology, visual inspection gradually develops into a modern integrated science and technology, and with its high speed, high precision and non-contact advantages, has been applied increasingly widely in the field of much industrial detection[1,2]. Line array camera is selected in visual detecting experimental device in this paper to acquire image acquisition. There is a necessary condition when the linear array camera acquire accurate images, first, the motion plane of the measured work piece and camera lens plane must be maintained a certain parallelism, i.e., the camera must be vertical with the table surface, so that it can guarantee the collected image without distortion[3,4]. Therefore it is necessary that the profile frame which is fixed camera components should be done the finite element analysis of flexural distortion, to test the rationality of design, and to ensure the accuracy of the light mechanical and electrical integration detection system.

\section{Imaging system structure}

Imaging system design diagram of visual detecting experimental device is shown as Figure 1. CAD model of device components is established by ProE, as Figure 2 shows. Vertical 30 x 60 profiles is $600 \mathrm{~mm}$ long, lateral $40 \times 40$ profiles is $310 \mathrm{~mm}$ long. Firstly, some parts which have less impact of deformation on detection apparatus are simplified, and then the model is imported into ANSYS. Adding remote force to replace the impact produced by the camera components force on the lateral profile in the process of analysis, analyze the flexural deformation condition under the gravity and remote force of the related parts [5].

\section{Camera bracket finite element model}

The three parts of the camera bracket are using two kinds of materials, profiles are made of aluminum alloy, the density is $\rho=2770 \mathrm{~kg} / \mathrm{m}^{3}$, Modulus of elasticity is $E=71 \mathrm{GPa}$, poisson's ratio 
is $\gamma=0.33$, the connecting block material is 45 steel, the density is $\rho=7890 \mathrm{~kg} / \mathrm{m}^{3}$, the density

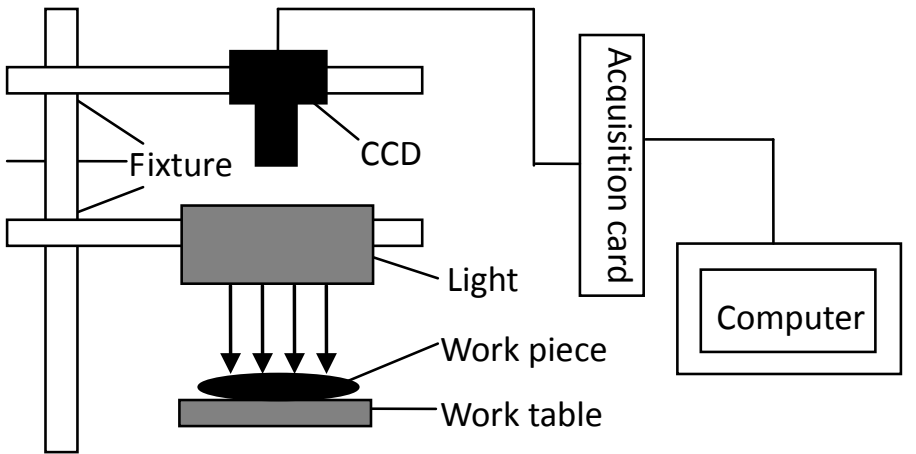

Fig. 1 Detection system schematic

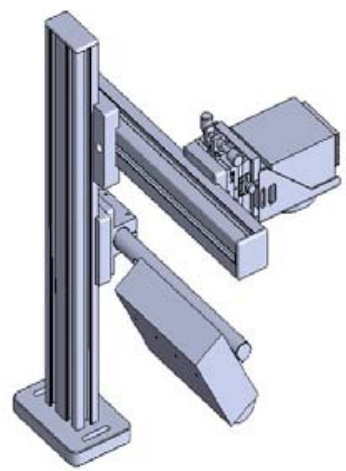

Fig. 2 Profiles assembly

is $E=210 G P a$, poisson's ratio is $\gamma=0.269$. When mesh the model, select a unit size to divide and get the solution. Then reduce the cell size by half again after the partition and get the solution. Comparison the two groups of the solution, if the results are basically consistent, then choose the last unit size in order to improve the computing speed. If the result of two sets is larger, then on the second unit size shrink again, mesh again and gat the solution by comparison $[6,7]$. This paper finally determines that the unit size is $5 \mathrm{~mm}$, and gets 92662 elements, 492877 nodes. Mesh diagram is shown in figure 3.

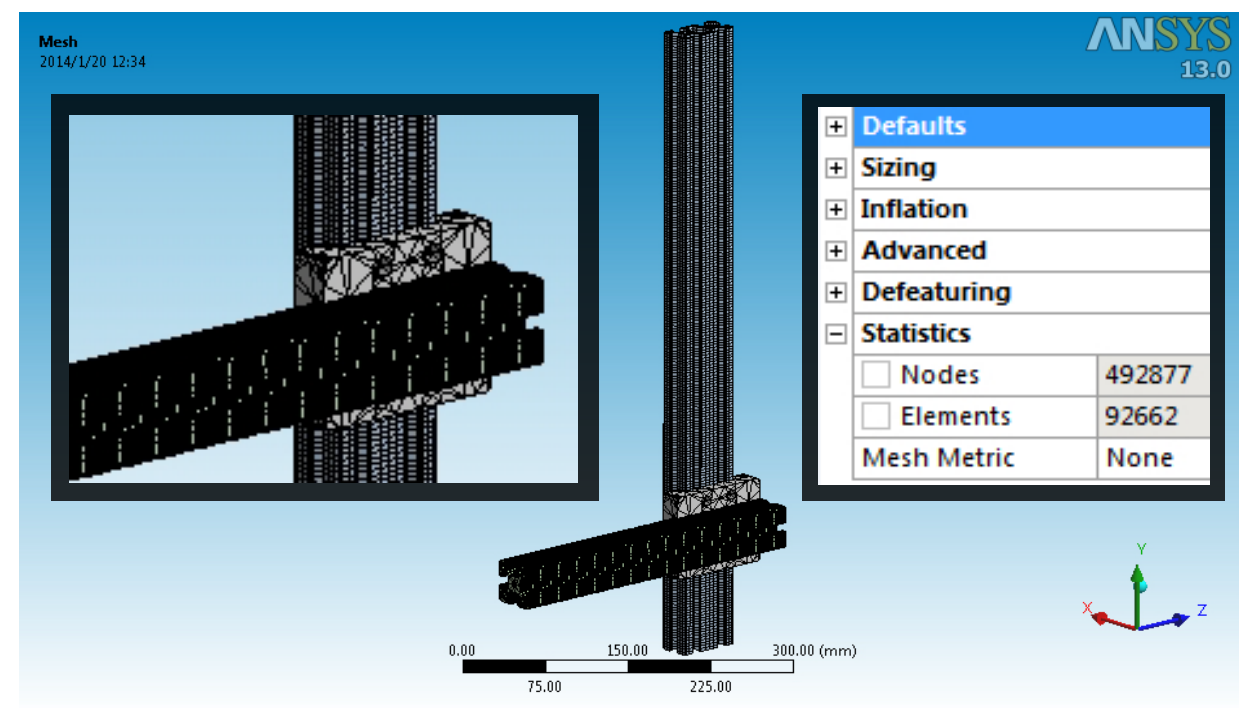

Fig.3 Stent mesh diagram

\section{Flexural deformation analysis of camera bracket}

Standard acceleration of gravity is applied on the $\mathrm{Z}$ direction in the whole model, normal constraint is applied to the profile face, and the fixed constraint is applied to the junction, and the camera, fine-tuning device and the connecting board are added into the finite element model by the quality unit. Use ANSYS Workbench to analyze and calculate many times, and obtain the flexure deformation of transverse section, according to the different install position of camera components. Deformation data is as shown in table 1 . 
From the data in Table 1, it is concluded that when the vertical height of transverse sectional is unchanged, with the increase of projection amount of the distance from camera to vertical profile. The maximum deflection of transverse section deformation is in rapid increase. When the projection amount of the distance from camera to vertical profile is not changed, with the increase of the transverse section height, Maximum deformation quantity of transverse sectional also increases quickly. The maximum can reach $0.13751 \mathrm{~mm}$. General trend of transverse sectional deformation is as shown in figure 4.

Table 1 Lateral section flexural deformation the camera assembly

Distance between camera components and vertical profile $(\mathrm{mm})$

\begin{tabular}{|c|c|c|c|c|c|c|c|c|}
\hline & 0 & 50 & 100 & 150 & 200 & 250 & 300 & 310 \\
\hline 100 & 0.021304 & 0.021084 & 0.022950 & 0.027073 & 0.035688 & 0.041643 & 0.053437 & 0.061440 \\
\hline 150 & 0.017753 & 0.018070 & 0.019125 & 0.022561 & 0.031344 & 0.041635 & 0.053457 & 0.061440 \\
\hline 200 & 0.021306 & 0.021379 & 0.022969 & 0.031791 & 0.036679 & 0.041677 & 0.062356 & 0.071693 \\
\hline 250 & 0.022381 & 0.022932 & 0.024516 & 0.034671 & 0.046893 & 0.053515 & 0.068882 & 0.079249 \\
\hline 300 & 0.027358 & 0.028179 & 0.032350 & 0.038303 & 0.052005 & 0.059558 & 0.076900 & 0.088523 \\
\hline 350 & 0.026530 & 0.030582 & 0.032101 & 0.038640 & 0.053147 & 0.061508 & 0.082002 & 0.090921 \\
\hline 400 & 0.030317 & 0.036085 & 0.043651 & 0.052209 & 0.061347 & 0.070840 & 0.092071 & 0.106110 \\
\hline 450 & 0.037939 & 0.039207 & 0.040619 & 0.056764 & 0.066831 & 0.077322 & 0.100670 & 0.116050 \\
\hline 500 & 0.040106 & 0.041306 & 0.043025 & 0.060519 & 0.071660 & 0.095189 & 0.108840 & 0.125500 \\
\hline 540 & 0.044399 & 0.045392 & 0.054975 & 0.066218 & 0.078410 & 0.104200 & 0.119190 & 0.137510 \\
\hline
\end{tabular}

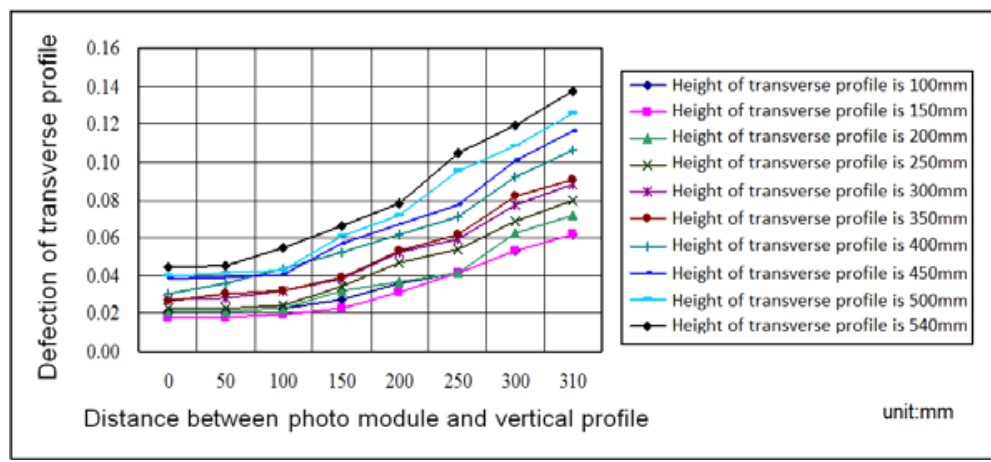

Fig. 4 Flexural deformation curve of ransverse section

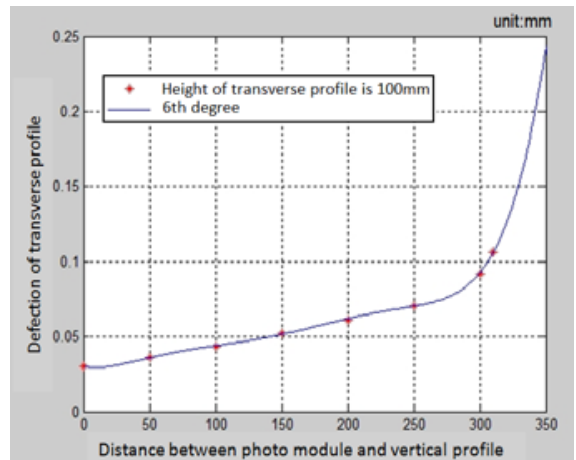

Fig. 5 Deflection fitting curve

Considering the light source installation space, camera work distance and deflection of the place transverse profiles connecting the camera, get the best installation location of camera in the space: the vertical height of transverse profile is $400 \mathrm{~mm}$, the distance between camera components and vertical profile is $200 \mathrm{~mm}$. at this position, in order to obtain the deflection at any point, use the polyfit function of Matlab to realize the least squares curve fitting for the deflection of transverse profile[8]. The fitting curve is as shown in figure 5, the position of remote stress is shown as figure 6 , and deflection deformation of transverse profile is shown in figure7. 


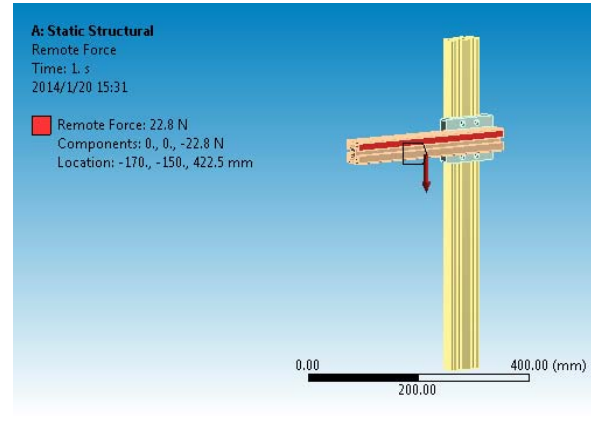

Fig. 6 Position of emote sress

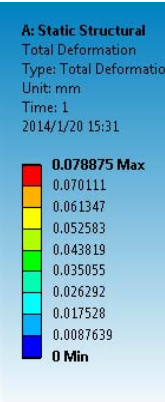

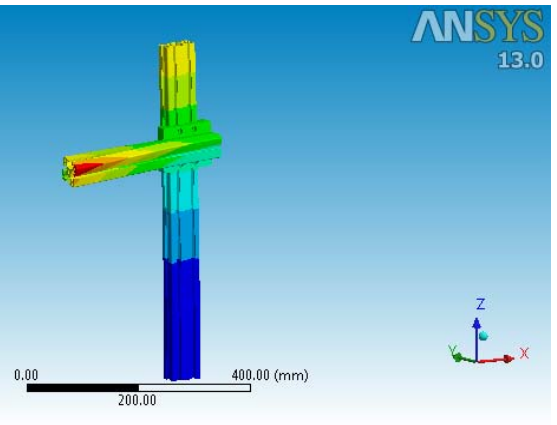

Fig. 7 Deformation

Shown as Figure 7, when the camera is installed at this position, the profile deflection deformation is $0.061347 \mathrm{~mm}$, but the accuracy of the main body of the device is $0.01 \mathrm{~mm}$, so the position state of the camera must be fine tuning, otherwise it will seriously affect the quality of image, cause the image distortion and inaccurate results. Therefore, according to the amount of deformation, adjust the camera position in space with the fine-tuning device installed, making the camera mirror and the work surface maintain a certain degree of parallelism, and reducing the measuring error.

\section{Conclusion}

According to the flexural deformation of the finite element analysis, use vernier device to adjust, so that the linear array camera CCD plane and the table surface can maintain a certain parallelism, and ensure the linear array camera capture the image of high quality. It provides a good hardware foundation for reducing the measurement error of the system.

\section{Reference}

[1] S.H.Sun. The key technology of visual measurement and its applications in automatic measurement . Tianjing: Doctoral Dissertation of Tianjin University, 2007 $\square$ pp.11-13

[2] C.F. The application of machine vision in industrial inspection. Automation panorama $\square$ 04 (2007)46-48.

[3] C.L.Xia. Overview of online detection method for surface quality of plate. Industrial measurement, 20 (2010) 1-5.

[4] L.Zhou. Study on cotton seed testing and sorting system . Beijing: Master degree thesis of China Agricultural University, 2010, pp.33-34

[5] Y.J.Shang. The finite element method and the ANSYS Application Guide . Beijing: Tsinghua University press $\square 2005 \square$ pp.23-25

[6] P.A.Du.. The finite element method- theory, modeling and application . Beijing: National

Defense Industry Press, $2011 \square$ pp.46-47

[7] B.L. ANSYS Workbench design and simulation and optimization . Beijing: Tsinghua University press, 2008, pp.56-58

[8] P.Zhou. Matlab numerical analysis . Beijing: Machinery Industry Press, 2009, pp.72 